\title{
Infinitely many positive solutions for a double phase problem
}

\author{
Bei-Lei Zhang ${ }^{1}$, Bin Ge1 and Gang-Ling Hou²*
}

"Correspondence:

hougl@hrbeu.edu.cn

${ }^{2}$ College of Aerospace and Civil

Engineering, Harbin Engineering

University, Harbin, 150001, P.R. China

Full list of author information is

available at the end of the article

\begin{abstract}
This paper is concerned with the existence of infinitely many positive solutions to a class of double phase problem. By variational methods and the theory of the Musielak-Orlicz-Sobolev space, we establish the existence of infinitely many positive solutions whose $W_{0}^{1, H}(\Omega)$-norms and $L^{\infty}$-norms tend to zero under suitable hypotheses about nonlinearity.
\end{abstract}

MSC: 35J60; 03H10; 35D05

Keywords: Double phase operator; Multiple solutions; Variational methods

\section{Introduction and main results}

The study of differential equations and variational problems with double phase operator is a new and interesting topic. Such interest is widely justified by many physical examples, such as elasticity, strongly anisotropic materials and Lavrentiev's phenomenon (e.g., see Refs. [1-4]). More precisely, their research is related to the following energy functional:

$$
u \mapsto \int_{\Omega}\left(|\nabla u|^{p}+a(x)|\nabla u|^{q}\right) d x
$$

where the integrand switches two different elliptic behaviors. In [4], energies of the form (1.1) are used in the context of homogenization and elasticity and $a(\cdot)$ drives the geometry of a composite of two different materials with hardening powers $p$ and $q$.

In the past the problem of existence and multiplicity of nontrivial solutions for double phase problems driven by the double phase operator was studied in the context of Dirichlet boundary value problems. We mention the work of Perera-Squassina [5], PapageorgiouRadulescu-Repovs [6, 7], Cencelj-Radulescu-Repovs [8], Zhang-Radulescu [9], Radulescu [10], Ge-Wang-Lu[11], Ge-Chen [12], Ge-Lv-Lu [13], Liu-Dai [14, 15], Colasuonno-Squassina [16] and the references therein. On the regularity for minimizers of variational problems and solutions of differential equations with double phase operator, we refer to [17-20], respectively, and the references therein.

(c) The Author(s) 2020. This article is licensed under a Creative Commons Attribution 4.0 International License, which permits use, sharing, adaptation, distribution and reproduction in any medium or format, as long as you give appropriate credit to the original author(s) and the source, provide a link to the Creative Commons licence, and indicate if changes were made. The images or other third party material in this article are included in the article's Creative Commons licence, unless indicated otherwise in a credit line to the material. If material is not included in the article's Creative Commons licence and your intended use is not permitted by statutory regulation or exceeds the permitted use, you will need to obtain permission directly from the copyright holder. To view a copy of this licence, visit http://creativecommons.org/licenses/by/4.0/. 
The aim of this paper is to obtain infinitely many distinct positive solutions for the following double phase problem:

$$
\begin{cases}-\operatorname{div}\left(|\nabla u|^{p-2} \nabla u+a(x)|\nabla u|^{q-2} \nabla u\right)=f(x, u), & \text { in } \Omega, \\ u=0, & \text { on } \partial \Omega,\end{cases}
$$

where $\Omega$ is a smooth bounded domain in $\mathbb{R}^{N}(N \geq 2), 1<p<q<N$,

$$
\frac{q}{p}<1+\frac{1}{N}, \quad a: \Omega \rightarrow[0,+\infty) \text { is Lipschitz continuous }
$$

and $f: \Omega \times \mathbb{R} \rightarrow \mathbb{R}$ satisfy Carathéodory condition and there exists $t_{0}>0$ such that $\sup _{t \in\left[0, t_{0}\right]} f(\cdot, t) \in L^{\infty}(\Omega)$.

In the past decade, many authors considered the existence and multiplicity of solutions of $(P)$. For example, Liu and Dai [14] got one sign-changing ground state solution for problem $(P)$ using the Nehari manifold method. Additionally, Liu and Dai in [15] also obtained the existence of at least three ground state solutions of $(P)$ by using the strong maximum principle. In a recent paper [12], Ge and Chen obtained the same result as in [14] for problem $(P)$ under more general assumptions on $f$. In [16], by using the fountain and dual theorem with Cerami condition, we obtained some existence of infinitely many solutions for the above problem under some weaker assumptions on $f$. The aim of the present paper is to establish the existence of infinitely many distinct positive solutions for problem $(P)$ under suitable oscillatory assumptions on the nonlinear term $f$ at zero.

In order to state the main result of this paper, let us introduce the following assumptions for problem $(P)$ :

$\left(h_{1}\right)$ There are two sequences $\left\{a_{k}\right\}_{k=1}^{\infty},\left\{b_{k}\right\}_{k=1}^{\infty}$ such that $0<a_{k}<b_{k}, \lim _{k \rightarrow+\infty} b_{k}=0$, and

$$
\int_{0}^{a_{k}} f(x, s) d s=\sup _{t \in\left[a_{k}, b_{k}\right]} \int_{0}^{t} f(x, s) d s
$$

for almost all $x \in \Omega$ and $k \in N$.

$\left(h_{2}\right)$ There exists a sequence $\left\{c_{k}\right\}_{k=1}^{\infty} \subset\left(0, b_{k}\right]$ such that

$$
\underset{x \in \Omega}{\operatorname{essinf}} \int_{0}^{c_{k}} f(x, s) d s>0
$$

We are now in the position to state our main results.

Theorem 1.1 Suppose that $f(x, 0)=0$ and $\left(h_{1}\right)-\left(h_{2}\right)$ hold. Then there exists a sequence $\left\{u_{k}\right\} \subset W_{0}^{1, H}(\Omega)$ of positive weak solutions of $(P)$ such that

$$
\begin{aligned}
& \lim _{k \rightarrow+\infty}\left(\int_{\Omega}\left(\frac{1}{p}\left|\nabla u_{k}\right|^{p}+\frac{a(x)}{q}\left|\nabla u_{k}\right|^{q}\right) d x-\int_{\Omega} F\left(x, u_{k}(x)\right) d x\right)=0 \text { and } \\
& \lim _{k \rightarrow+\infty}\left\|u_{k}\right\|=0,
\end{aligned}
$$

$(\|\cdot\|$ is defined in $(2.1))$. 
The rest of this paper is organized as follows. In Sect. 2, we present some necessary preliminary knowledge on space $W_{0}^{1, H}(\Omega)$. In Sect. 3, we establish the variational framework associated with problem $(P)$, and we complete the proofs of Theorem 1.1.

\section{Preliminaries}

In order to discuss problem $(P)$, we need some facts on space $W_{0}^{1, H}(\Omega)$ which are called Musielak-Orlicz-Sobolev space. For this reason, we will recall some properties involving the Musielak-Orlicz spaces, which can be found in [16, 21-23] and the references therein.

Denote by $N(\Omega)$ the set of all generalized $N$-function. For $1<p<q$ and $0 \leq a(\cdot) \in L^{1}(\Omega)$, we define

$$
H(x, t)=t^{p}+a(x) t^{q}, \quad \forall(x, t) \in \Omega \times[0,+\infty) .
$$

It is clear that $H \in N(\Omega)$ is a locally integrable and

$$
H(x, 2 t) \leq 2^{q} H(x, t), \quad \forall(x, t) \in \Omega \times[0,+\infty),
$$

which is called condition $\left(\Delta_{2}\right)$.

The Musielak-Orlicz space $L^{H}(\Omega)$ is defined by

$$
L^{H}(\Omega)=\left\{u: \Omega \rightarrow \mathbb{R} \text { measurable }: \int_{\Omega} H(x,|u|) d x<+\infty\right\},
$$

endowed with the Luxemburg norm

$$
|u|_{H}=\inf \left\{\lambda>0: \int_{\Omega} H\left(x,\left|\frac{u}{\lambda}\right|\right) d x \leq 1\right\}
$$

The Musielak-Orlicz-Sobolev space $W^{1, H}(\Omega)$ is defined by

$$
W^{1, H}(\Omega)=\left\{u \in L^{H}(\Omega):|\nabla u| \in L^{H}(\Omega)\right\},
$$

and it is equipped with the norm

$$
\|u\|=|u|_{H}+|\nabla u|_{H} .
$$

We denote by $W_{0}^{1, H}(\Omega)$ the completion of $C_{0}^{\infty}(\Omega)$ in $W^{1, H}(\Omega)$. With these norms, the spaces $W_{0}^{1, H}(\Omega)$ and $W^{1, H}(\Omega)$ are separable reflexive Banach spaces; see [16, Proposition 2.18] for the details. Moreover, thanks to (1.2) and [16, Proposition 2.18] we can see that

$$
\|u\|=|\nabla u|_{H}, \quad \forall u \in W_{0}^{1, H}(\Omega)
$$

is an equivalent norm on $W_{0}^{1, H}(\Omega)$.

Furthermore, we have the following embedding theorem. 
Proposition 2.1 ([16, Proposition 2.15])

(1) If $1 \leq s \leq p$, then the embedding from $W_{0}^{1, H}(\Omega)$ to $W_{0}^{1, s}(\Omega)$ is continuous.

(2) If $1 \leq s \leq p^{*}=\frac{N p}{N-p}$, then the embedding from $W_{0}^{1, H}(\Omega)$ to $L^{s}(\Omega)$ is continuous. In particular, if $s \in\left[1, p^{*}\right)$, then the embedding $W_{0}^{1, H}(\Omega) \hookrightarrow L^{s}(\Omega)$ is compact.

From Liu-Dai [14, Proposition 2.1]) we directly obtain

$$
\begin{aligned}
& \|u\|^{q} \leq \int_{\Omega}\left(|\nabla u|^{p}+a(x)|\nabla u|^{q}\right) d x \leq\|u\|^{p}, \quad \text { if }\|u\| \leq 1 ; \\
& \|u\|^{p} \leq \int_{\Omega}\left(|\nabla u|^{p}+a(x)|\nabla u|^{q}\right) d x \leq\|u\|^{q}, \quad \text { if }\|u\| \geq 1,
\end{aligned}
$$

for any $u \in W_{0}^{1, H}(\Omega)$.

From now on, we denote by $E$ the space $W_{0}^{1, H}(\Omega)$. In order to discuss the problem $(P)$, we need to define a functional in $E$ :

$$
J(u)=\int_{\Omega}\left(\frac{1}{p}|\nabla u|^{p}+\frac{a(x)}{q}|\nabla u|^{q}\right) d x .
$$

We know that $J \in C^{1}(E, \mathbb{R})$ and double phase operator $-\operatorname{div}\left(|\nabla u|^{p-2} \nabla u+a(x)|\nabla u|^{q-2} \nabla u\right)$ is the derivative operator of $J$ in the weak sense. We denote $L=J^{\prime}: E \rightarrow E^{*}$, then

$$
\langle L(u), v\rangle=\int_{\Omega}\left(|\nabla u|^{p-2} \nabla u \cdot \nabla v+a(x)|\nabla u|^{q-2} \nabla u \cdot \nabla v\right) d x
$$

for all $u, v \in E$. Here $E^{*}$ denotes the dual space of $E$ and $\langle\cdot, \cdot\rangle$ denotes the pairing between $E$ and $E^{*}$. Then we have the following result.

Proposition 2.2 ([14, Proposition 3.1]) If $L$ is as above, then

(1) $L: E \rightarrow E^{*}$ is a continuous, bounded and strictly monotone operator;

(2) $L: E \rightarrow E^{*}$ is a mapping of type $(S)_{+}$, i.e., if $u_{n} \rightarrow u$ in $E$ and $\lim \sup _{n \rightarrow+\infty}\left\langle L\left(u_{n}\right)-L(u), u_{n}-u\right\rangle \leq 0$, implies $u_{n} \rightarrow u$ in $E$;

(3) $L: E \rightarrow E^{*}$ is a homeomorphism.

\section{Variational setting and the proof of Theorem 1.1}

To prove our Theorem 1.1, we recall the variational setting corresponding to the problem $(P)$.

We observe that problem $(P)$ has a variational structure, and as a matter of fact, its solutions can be searched as critical points of the energy functional $\varphi: E \rightarrow \mathbb{R}$ defined as follows:

$$
\varphi=\Phi(u)-\Psi(u)
$$

where

$$
\Phi(u)=\int_{\Omega}\left(\frac{1}{p}|\nabla u|^{p}+\frac{a(x)}{q}|\nabla u|^{q}\right) d x
$$


and $\Psi(u)=\int_{\Omega} F(x, u(x)) d x$. Thus, in [14], it is shown that $\Phi(u)$ is a Gâteaux differentiable functional in $E$ whose derivative is given by

$$
\left\langle\Phi^{\prime}(u), v\right\rangle=\int_{\Omega}\left(|\nabla u|^{p-2} \nabla u \cdot \nabla v+a(x)|\nabla u|^{q-2} \nabla u \cdot \nabla v\right) d x
$$

for all $v \in E$. Finally, $\Phi(u)$ is weakly lower semi-continuous and coercive. Moreover, standard arguments show that $\Psi$ is a well defined and continuously Gâteaux differentiable functional whose Gâteaux derivative

$$
\left\langle\Psi^{\prime}(u), v\right\rangle=\int_{\Omega} f(x, u) v d x
$$

for all $v \in E$.

Definition 3.1 We say that $u \in E$ is a weak solution of $(P)$ if

$$
\int_{\Omega}\left(|\nabla u|^{p-2} \nabla u \cdot \nabla v+a(x)|\nabla u|^{q-2} \nabla u \cdot \nabla v\right) d x=\int_{\Omega} f(x, u) v d x,
$$

for all $v \in E$.

Next, we will prove Theorem 1.1 by virtue of some idea due to Kristaly, Morosanu and Tersian [24], where the infinitely many homoclinic solutions for a $p$-Laplace equation was obtained. Firstly, by our assumptions on $f$, there exist $d_{0}>0$ and $t_{0}>0$ such that $|f(x, t)| \leq$ $d_{0}$, for every $t \in\left[0, t_{0}\right]$ and a.e. $x \in \Omega$. Without loss of generality, we suppose that, for every $k \in N, b_{k} \leq t_{0}$, where $b_{k}$ is from $\left(h_{1}\right)$. Define

$$
\tilde{f}(x, t)= \begin{cases}0, & \text { if } t \leq 0 \\ f(x, u), & \text { if } 0<t \leq t_{0} \\ f\left(x, t_{0}\right), & \text { if } t>t_{0}\end{cases}
$$

Thus, we have

$$
|\widetilde{f}(x, t)| \leq d_{0}, \quad \forall t \in \mathbb{R} \text { and a.e. } x \in \Omega
$$

Now, we consider the following problem:

$$
\begin{cases}-\operatorname{div}\left(|\nabla u|^{p-2} \nabla u+a(x)|\nabla u|^{q-2} \nabla u\right)=\tilde{f}(x, u), & \text { in } \Omega, \\ u=0, & \text { on } \partial \Omega,\end{cases}
$$

Hence, the weak solutions of (3.4) are the critical points of the functional

$$
\widetilde{\varphi}(u)=\Phi(u)-\int_{\Omega} \widetilde{F}(x, u(x)) d x,
$$

where $\widetilde{F}(x, u)=\int_{0}^{u} \widetilde{f}(x, s) d s$.

By (3.3), it is easy to see that $\tilde{\varphi}$ is well defined, weakly sequentially lower semi-continuous and Gâteaux differentiable in $E$. For every fixed $k \in N$, consider the set $S_{k}=\{u \in E: u(x) \neq$ 0 and $0 \leq u(x) \leq b_{k}$ a.e. $\left.x \in \Omega\right\}$. 
Lemma 3.2 For every $k \in N$, the functional $\widetilde{\varphi}$ is bounded from below on $S_{k}$ and its infimum $m_{k}$ on $S_{k}$ is attained at $u_{k} \in S_{k}$.

Proof For every $k \in N$, we obtain, for any every $u \in S_{k}$,

$$
\begin{aligned}
\widetilde{\varphi}(u) & =\int_{\Omega}\left(\frac{1}{p}|\nabla u|^{p}+\frac{a(x)}{q}|\nabla u|^{q}\right) d x-\int_{\Omega} \widetilde{F}(x, u) d x \\
& \geq-\int_{\Omega} \widetilde{F}(x, u) d x \\
& \geq-d_{0} b_{k}|\Omega| .
\end{aligned}
$$

This means that $\widetilde{\varphi}$ is bounded from below on $S_{k}$.

Moreover, it is clear that $S_{k}$ is convex and closed, thus weakly closed in $E$. Let $m_{k}=$ $\inf _{u \in S_{k}} \widetilde{\varphi}(u)$, and $\left\{u_{n}\right\}$ be a sequence in $S_{k}$ such that $m_{k} \leq \widetilde{\varphi}\left(u_{n}\right) \leq m_{k}+\frac{1}{n}$ for all $n \in N$. Then, if $\left\|u_{n}\right\| \leq 1$, we have done it, otherwise, we have

$$
\frac{1}{q}\left\|u_{n}\right\|^{p} \leq m_{k}+1+d_{0} b_{k}|\Omega|, \quad \forall n \in N
$$

From this, we deduce that $\left\{u_{n}\right\}$ is bounded in $E$. So, up to a subsequence, $\left\{u_{n}\right\}$ weakly converges to some $u_{k} \in S_{k}$. At this point, we obtain $\widetilde{\varphi}\left(u_{k}\right)=m_{k}$ in view of the weakly sequentially lower semi-continuity of $\widetilde{\varphi}$. This completes the proof of the lemma.

Lemma 3.3 For every $k \in N, 0 \leq u_{k}(x) \leq a_{k}$ a.e. $x \in \Omega$.

Proof Let $T=\left\{x \in \Omega: a_{k}<u_{k}(x) \leq b_{k}\right\}$ and assume that meas $(T)>0$. Define the function $h(t)=\min \left\{t^{+}, a_{k}\right\}$ and $v_{k}=h\left(u_{k}\right)$, where $t^{+}=\max \{0, t\}$. It is obvious that $h$ is continuous in $E$. Moreover, $0 \leq v_{k}(x) \leq a_{k}$ for a.e. $x \in \Omega$. Consequently, $v_{k} \in S_{k}$ and

$$
v_{k}(x)= \begin{cases}u_{k}(x), & \text { if } x \in \Omega \backslash T, \\ a_{k}, & \text { if } x \in T .\end{cases}
$$

Moreover, we have

$$
\begin{aligned}
\widetilde{\varphi}\left(v_{k}\right)-\widetilde{\varphi}\left(u_{k}\right)= & \int_{\Omega}\left(\frac{1}{p}\left|\nabla v_{k}\right|^{p}+\frac{a(x)}{q}\left|\nabla v_{k}\right|^{q}\right) d x-\int_{\Omega} \widetilde{F}\left(x, v_{k}\right) d x \\
& -\int_{\Omega}\left(\frac{1}{p}\left|\nabla u_{k}\right|^{p}+\frac{a(x)}{q}\left|\nabla u_{k}\right|^{q}\right) d x+\int_{\Omega} \widetilde{F}\left(x, u_{k}\right) d x \\
= & \int_{T}\left(\frac{1}{p}\left|\nabla v_{k}\right|^{p}+\frac{a(x)}{q}\left|\nabla v_{k}\right|^{q}\right) d x-\int_{T} \widetilde{F}\left(x, v_{k}\right) d x \\
& -\int_{T}\left(\frac{1}{p}\left|\nabla u_{k}\right|^{p}+\frac{a(x)}{q}\left|\nabla u_{k}\right|^{q}\right) d x+\int_{T} \widetilde{F}\left(x, u_{k}\right) d x \\
= & 0-\int_{T}\left(\frac{1}{p}\left|\nabla u_{k}\right|^{p}+\frac{a(x)}{q}\left|\nabla u_{k}\right|^{q}\right) d x \\
& -\int_{T}\left(\widetilde{F}\left(x, a_{k}\right)-\widetilde{F}\left(x, u_{k}\right)\right) d x .
\end{aligned}
$$


By $\left(h_{1}\right)$, we have $\int_{T}\left(\widetilde{F}\left(x, a_{k}\right)-\widetilde{F}\left(x, u_{k}\right)\right) d x \geq 0$. Consequently, every term of the expression $\widetilde{\varphi}\left(v_{k}\right)-\widetilde{\varphi}\left(u_{k}\right)$ is non-positive. On the other hand, since $v_{k} \in S_{k}$, then $\widetilde{\varphi}\left(v_{k}\right) \geq \widetilde{\varphi}\left(u_{k}\right)=$ $\inf _{v \in S_{k}} \widetilde{\varphi}(v)$. So, every term in $\widetilde{\varphi}\left(v_{k}\right)-\widetilde{\varphi}\left(u_{k}\right)$ should be zero. In particular,

$$
\int_{T}\left(\frac{1}{p}\left|\nabla u_{k}\right|^{p}+\frac{a(x)}{q}\left|\nabla u_{k}\right|^{q}\right) d x=\int_{T}\left(\widetilde{F}\left(x, a_{k}\right)-\widetilde{F}\left(x, u_{k}\right)\right) d x=0,
$$

which implies that meas $(T)=0$.

Lemma 3.4 For every $k \in N, u_{k}$ is a local minimum point of $\widetilde{\varphi}$ in $E$.

Proof Let $T^{\prime}=\left\{x \in \Omega: u(x) \notin\left(0, a_{k}\right]\right\}$. Set $v=h(u)$, then we observe that

$$
\int_{v}^{u} \widetilde{f}(x, s) d s=0, \quad \forall x \in \Omega \backslash T^{\prime}
$$

Moreover, if $x \in T^{\prime}$, then one has the following three cases:

(a) When $u(x)<0$, then $\int_{v}^{u} \widetilde{f}(x, s) d s=0$;

(b) When $a_{k}<u(x) \leq b_{k}$, then from $\left(h_{1}\right)$, we deduce that $\int_{v}^{u} \widetilde{f}(x, s) d s \leq 0$;

(c) When $u(x)>b_{k}$, then $\int_{v}^{u} \widetilde{f}(x, s) d s=\int_{a_{k}}^{u} \tilde{f}(x, s) d s \leq \int_{a_{k}}^{u} d_{0} d s=d_{0}\left(u(x)-a_{k}\right)$.

Let $p^{*} \geq \theta+1>q$ for every $x \in \Omega$ and fix it, then the constant

$$
C_{0}=\sup _{s \geq b_{k}} \frac{d_{0}\left(s-a_{k}\right)}{\left(s-a_{k}\right)^{\theta+1}}
$$

is finite, we have, for a.e. $x \in \Omega, \int_{v}^{u} \widetilde{f}(x, s) d s \leq C_{0}|u(x)-v(x)|^{\theta+1}$. Then, using Propositions 2.1, we have

$$
\int_{\Omega} \int_{v}^{u} \widetilde{f}(x, s) d s d x \leq C_{0} c^{\theta+1}\|u-v\|^{\theta+1},
$$

where $c$ is the embedding constant of $E \hookrightarrow L^{\theta+1}(\Omega)$. We have

$$
\begin{aligned}
\widetilde{\varphi}(u)-\widetilde{\varphi}(v)= & \int_{\Omega}\left(\frac{1}{p}|\nabla u|^{p}+\frac{a(x)}{q}|\nabla u|^{q}\right) d x-\int_{\Omega} \widetilde{F}(x, u) d x \\
& -\int_{\Omega}\left(\frac{1}{p}|\nabla v|^{p}+\frac{a(x)}{q}|\nabla v|^{q}\right) d x+\int_{\Omega} \widetilde{F}(x, v) d x \\
= & \int_{T^{\prime}}\left(\frac{1}{p}|\nabla u|^{p}+\frac{a(x)}{q}|\nabla u|^{q}\right) d x-\int_{T^{\prime}} \widetilde{F}(x, u) d x \\
& -\int_{T^{\prime}}\left(\frac{1}{p}|\nabla v|^{p}+\frac{a(x)}{q}|\nabla v|^{q}\right) d x+\int_{T^{\prime}} \widetilde{F}(x, v) d x \\
= & \int_{T^{\prime}}\left(\frac{1}{p}|\nabla u|^{p}+\frac{a(x)}{q}|\nabla u|^{q}\right) d x-\int_{T^{\prime}} \int_{v}^{u} \widetilde{f}(x, s) d s d x \\
= & \int_{\Omega}\left(\frac{1}{p}|\nabla(u-v)|^{p}+\frac{a(x)}{q}|\nabla(u-v)|^{q}\right) d x-\int_{T^{\prime}} \int_{a_{k}}^{u} f(x, s) d x \\
\geq & \int_{\Omega}\left(\frac{1}{p}|\nabla(u-v)|^{p}+\frac{a(x)}{q}|\nabla(u-v)|^{q}\right) d x-C_{0} c^{\theta+1}\|u-v\|^{\theta+1} .
\end{aligned}
$$


On the other hand, by $v \in S_{k}$, we have

$$
\widetilde{\varphi}(v) \geq \widetilde{\varphi}\left(u_{k}\right)
$$

Using (2.1) and (3.9) in (3.8), we obtain

$$
\begin{aligned}
\widetilde{\varphi}(u) \geq & \tilde{\varphi}\left(u_{k}\right)+\int_{\Omega}\left(\frac{1}{p}|\nabla(u-v)|^{p}+\frac{a(x)}{q}|\nabla(u-v)|^{q}\right) d x \\
& -C_{0} c^{\theta+1}\|u-v\|^{\theta+1} \\
\geq & \tilde{\varphi}\left(u_{k}\right)+\frac{1}{q}\|u-v\|^{\tau}-C_{0} c^{\theta+1}\|u-v\|^{\theta+1} \\
= & \widetilde{\varphi}\left(u_{k}\right)+\|u-v\|^{\tau}\left(\frac{1}{q}-C_{0} c^{\theta+1}\|u-v\|^{\theta+1-\tau}\right),
\end{aligned}
$$

where $\tau=p(q)$ when $\|u-v\| \geq 1(\leq 1)$.

Since $h$ is continuous, there exists $\delta>0$ such that, for every $u \in E$ with

$$
\left\|u-u_{k}\right\|<\delta, \quad\|u-v\| \leq \frac{1}{q C_{0} c^{\theta+1}},
$$

which implies that $u_{k}$ is a local minimum of $\tilde{\varphi}$.

Remark 3.5 Using Theorem 3.3 of [15], we have $u_{k}>0$ on $S_{k}$.

Lemma 3.6 For every $k \in N, m_{k}<0$ and $\lim _{k \rightarrow+\infty} m_{k}=0$.

Proof In view of $\left(h_{2}\right)$, one easily deduces $c_{k} \in S_{k}$. Hence,

$$
\begin{aligned}
m_{k} \leq \widetilde{\varphi}\left(c_{k}\right) & =-\int_{\Omega} \widetilde{F}\left(x, c_{k}\right) d x \\
& =-\int_{\Omega} F\left(x, c_{k}\right) d x \\
& =-\int_{\Omega} \int_{0}^{c_{k}} f\left(x, c_{k}\right) d x \\
& <0 .
\end{aligned}
$$

Now we will prove that $\lim _{k \rightarrow+\infty} m_{k}=0$.

As a result of Lemma 3.2, for every $k \in N$ and $u_{k} \in S_{k}$, we obtain

$$
\begin{aligned}
m_{k} & =\widetilde{\varphi}\left(u_{k}\right) \\
& =\int_{\Omega}\left(\frac{1}{p}\left|\nabla u_{k}\right|^{p}+\frac{a(x)}{q}\left|\nabla u_{k}\right|^{q}\right) d x-\int_{\Omega} \widetilde{F}\left(x, u_{k}\right) d x \\
& \geq-d_{0} \operatorname{meas}(\Omega) b_{k} .
\end{aligned}
$$

Since $\lim _{k \rightarrow+\infty} b_{k}=0$, we have $\lim _{k \rightarrow+\infty} m_{k} \geq 0$. Note that $m_{k}<0$, hence $\lim _{k \rightarrow+\infty} m_{k}=$ 0 . 
Now, we are ready to prove Theorem 1.1.

Proof of Theorem 1.1 Since $u_{k}$ are local minima of $\widetilde{\varphi}$, they are critical points of $\widetilde{\varphi}$, thus weak solutions of $(P)$. In view of Lemma 3.3, we can deduce that there are infinitely many distinct $u_{k}$ with $\lim _{k \rightarrow+\infty}\left|u_{k}\right|_{\infty}=0$. Moreover, we have

$$
\begin{aligned}
\left\|u_{k}\right\|^{\tau} & \leq \int_{\Omega}\left(\frac{1}{p}\left|\nabla u_{k}\right|^{p}+\frac{a(x)}{q}\left|\nabla u_{k}\right|^{q}\right) d x \\
& =m_{k}+\int_{\Omega} \widetilde{F}\left(x, u_{k}\right) d x \\
& \leq m_{k}+\operatorname{meas}(\Omega) d_{0} b_{k},
\end{aligned}
$$

where $\tau=p(q)$ when $\left\|u_{k}\right\| \geq 1(\leq 1)$. From this we conclude that $\lim _{k \rightarrow+\infty}\left\|u_{k}\right\|=0$. This completes the proof.

Example A simple example of a potential function satisfying hypotheses $\left(h_{1}\right)-\left(h_{2}\right)$ is

$$
f(x, t)= \begin{cases}\left(1+|x|^{p}\right)\left[(p+2) t^{p+1} \sin \frac{1}{t^{p}}-p t \cos \frac{1}{t^{p}}\right], & \text { if } t>0, \\ 0, & \text { otherwise }\end{cases}
$$

Proof It is easy to compute directly that

$$
F(x, t)= \begin{cases}\left(1+|x|^{p}\right) t^{p+2} \sin \frac{1}{t^{p}}, & \text { if } t>0 \\ 0, & \text { otherwise. }\end{cases}
$$

Let $\left\{a_{k}\right\},\left\{b_{k}\right\}$ and $\left\{c_{k}\right\}$ be three sequences such that

$$
a_{k}=\left(\frac{1}{(2 k+2) \pi}\right)^{\frac{1}{p}}, \quad b_{k}=\left(\frac{1}{(2 k+1.5) \pi}\right)^{\frac{1}{p}}, \quad c_{k}=\left(\frac{1}{(4 k+0.5) \pi}\right)^{\frac{1}{p}}
$$

for every $k \in N$. Then one easily deduces

$$
\int_{0}^{a_{k}} f(x, s) d s=\sup _{t \in\left[a_{k}, b_{k}\right]} \int_{0}^{t} f(x, s) d s
$$

and

$$
F\left(x, c_{k}\right) \geq 0 .
$$

So conditions $\left(h_{1}\right)-\left(h_{2}\right)$ have been satisfied.

\section{Acknowledgements}

The authors would like to thank the referees for their useful suggestions.

Funding

This work is supported by the National Key Research and Development Program of China (No. 2018YFC0310500), the National Natural Science Foundation of China (No. U1706227,11201095), the Fundamental Research Funds for the Central Universities, the Postdoctoral research startup foundation of Heilongjiang (No. LBH-Q14044), the Science Research Funds for Overseas Returned Chinese Scholars of Heilongjiang Province (No. LC201502). 


\section{Abbreviations}

Not applicable.

\section{Availability of data and materials}

Data sharing is not applicable to this article as no new data were created or analyzed in this study.

\section{Competing interests}

The authors declare that they have no competing interests.

\section{Authors' contributions}

The authors declare that the study was realized in collaboration with equal responsibility. All authors read and approved the final manuscript.

\section{Author details}

${ }^{1}$ School of Mathematical Sciences, Harbin Engineering University, Harbin, 150001, P.R. China. ${ }^{2}$ College of Aerospace and

Civil Engineering, Harbin Engineering University, Harbin, 150001, P.R. China.

\section{Publisher's Note}

Springer Nature remains neutral with regard to jurisdictional claims in published maps and institutional affiliations.

Received: 22 April 2020 Accepted: 12 August 2020 Published online: 28 August 2020

\section{References}

1. Zhikov, V.V.: Averaging of functionals of the calculus of variations and elasticity theory. Izv. Akad. Nauk SSSR, Ser. Mat. 50, 675-710 (1986)

2. Zhikov, V.V.: On Lavrentiev's phenomenon. Russ. J. Math. Phys. 3, 249-269 (1995)

3. Zhikov, V.V.: On some variational problems. Russ. J. Math. Phys. 5, 105-116 (1997)

4. Zhikov, V.V., Kozlov, S.M., Oleinik, O.A.: Homogenization of Differential Operators and Integral Functionals. Springer, Berlin (1994)

5. Perera, K., Squassina, M.: Existence results for double-phase problems via Morse theory. Commun. Contemp. Math. 20, 1750023 (2018)

6. Papageorgiou, N.S., Radulescu, V.D., Repovs, D.D.: Double-phase problems with reaction of arbitrary growth. Z. Angew. Math. Phys. 69, 108 (2018)

7. Papageorgiou, N.S., Repovs, D.D., Radulescu, V.D.: Double-phase problems and a discontinuity property of the spectrum. Proc. Am. Math. Soc. 147, 2899-2910 (2019)

8. Cencelj, M., Radulescu, V.D., Repovs, D.D.: Double phase problems with variable growth. Nonlinear Anal. 177, 270-287 (2018)

9. Zhang, Q.H., Radulescu, V.D.: Double phase anisotropic variational problems and combined effects of reaction and absorption terms. J. Math. Pures Appl. 118, 159-203 (2018)

10. Radulescu, V.D.: Isotropic and anisotropic double-phase problems: old and new. Opusc. Math. 39, 259-279 (2019)

11. Ge, B., Wang, L.Y., Lu, J.F.: On a class of double-phase problem without Ambrosetti-Robinowitz-type conditions. Appl. Anal. (2019, in press). https://doi.org/10.1080/00036811.2019.1679785

12. Ge, B., Chen, Z.Y.: Existence of infinitely many solutions for double phase problem with sign-changing potential. Rev. R. Acad. Cienc. Exactas Fís. Nat., Ser. A Mat. 113, 3185-3196 (2019)

13. Ge, B., Lv, D.L., Lu, J.F.: Multiple solutions for a class of double phase problem without the Ambrosetti-Rabinowitz conditions. Nonlinear Anal. 188, 294-315 (2019)

14. Liu, W.L., Dai, G.W.: Existence and multiplicity results for double phase problem. J. Differ. Equ. 265, 4311-4334 (2018)

15. Liu, W.L., Dai, G.W.: Three ground state solutions for double phase problem. J. Math. Phys. 59, 121503 (2018)

16. Colasuonno, F., Squassina, M.: Eigenvalues for double phase variational integrals. Ann. Mat. Pura Appl. 195, 1917-1959 (2016)

17. Baroni, P., Colombo, M., Mingione, G.: Harnack inequalities for double phase functionals. Nonlinear Anal. 121, 206-222 (2015)

18. Baroni, P., Colombo, M., Mingione, G.: Regularity for general functionals with double phase. Calc. Var. Partial Differ. Equ. 57, $62(2018)$

19. De Filippis, C., Palatucci, G.: Hölder regularity for nonlocal double phase equations. J. Differ. Equ. 267, 547-586 (2019)

20. Esposito, L., Leonetti, F., Mingione, G.: Sharp regularity for functionals with $(p, q)$ growth. J. Differ. Equ. 204, 5-55 (2004)

21. Musielak, J.: Orlicz Spaces and Modular Spaces. Lecture Notes in Math., vol. 1034. Springer, Berlin (1983)

22. Benkirane, A., Sidi El Vally, M.: Variational inequalities in Musielak-Orlicz-Sobolev spaces. Bull. Belg. Math. Soc. Simon Stevin 21,787-811 (2014)

23. Fan, X., Guan, C.X.: Uniform convexity of Musielak-Orlicz-Sobolev spaces and applications. Nonlinear Anal. 73, $163-175(2010)$

24. Kristaly, A., Morosanu, G., Tersian, S.: Quasilinear elliptic problems in $\mathbb{R}^{N}$ involving oscillatory nonlinearities. J. Differ. Equ. 235, 366-375 (2007) 